\title{
A formal semantics for isorecursive and equirecursive state abstractions
}

\section{Report}

\section{Author(s):}

Summers, Alexander J.; Drossopoulou, Sophia

Publication date:

2012

Permanent link:

https://doi.org/10.3929/ethz-a-007569992

\section{Rights / license:}

In Copyright - Non-Commercial Use Permitted

\section{Originally published in:}

Technical Report / ETH Zurich, Department of Computer Science 773 


\title{
A Formal Semantics for Isorecursive and Equirecursive State Abstractions
}

\author{
Alexander J. Summers ${ }^{1}$ and Sophia Drossopoulou ${ }^{2}$ \\ 1 ETH Zurich alexander.summers@inf.ethz.ch \\ 2 Imperial College London s.drossopoulou@imperial.ac.uk
}

\begin{abstract}
Most methodologies for static program verification support recursively-defined predicates in specifications, in order to reason about recursive data structures. Intuitively, a predicate instance represents the complete unrolling of its definition; this is the equirecursive interpretation. However, this semantics is unsuitable for static verification, when the recursion becomes unbounded. For this reason, most static verifiers supporting recursive definitions employ explicit folding and unfolding of recursive definitions (specified using ghost commands, or inferred). Such a semantics differentiates between, e.g., a predicate instance and its corresponding body, while providing a facility to map between the two; this is the isorecursive semantics. While this latter interpretation is usually implemented in practice, only the equirecursive semantics is typically treated in theoretical work.

In this paper we provide both an isorecursive and an equirecursive formal semantics for recursive definitions in the context of Chalice, a verification methodology based on implicit dynamic frames. We extend these assertion semantics to appropriate Hoare Logics, and prove the soundness of our definitions. The development of such formalisations requires addressing several subtle issues, regarding both the possibility of infinitelyrecursive definitions and the need for the isorecursive semantics to correctly reflect the restrictions that make it readily implementable. These questions are made more challenging still in the context of implicit dynamic frames, where the use of heap-dependent expressions provides further pitfalls for a correct formal treatment.
\end{abstract}

\section{Introduction}

For programs that manipulate recursively-defined data structures, it is impossible for a specification to directly list all of the locations and all the permissions [2] which are required to access the structure. For example, a method which computes the sum of the values in a linked-list will need to access a statically-unbounded number of heap locations to do so. To solve this specification problem, recursive abstract predicates [10] were introduced. The idea is that predicate definitions can be provided as part of a program's specification. The meaning of a predicate name is then defined in terms of an assertion (the predicate body), which may itself include instances of the same predicate. In this way, it is possible for a predicate instance to implicitly require permission to, 
e.g., every next field in a linked-list. The intuitive meaning of such a predicate symbol, is that it represents everything implied by the (recursive) unrolling of its definition; this is the equirecursive interpretation of the recursive definition.

However, static verifiers cannot reason directly in terms of an equirecursive semantics, since at verification time it is impossible to know when to stop unrolling such a definition. For this reason, most verifiers make use of ghost fold and unfold operations in the source code, to explicitly direct the tool when to unroll (or roll up) the definition of a predicate ${ }^{3}$. Such a treatment of recursive definitions differentiates between holding a predicate instance and holding its body, while providing a means by which the one can be explicitly exhanged for the other; this is the isorecursive interpretation.

The critical aspect of an isorecursive semantics is that it can be used as the basis for building static verifiers, while the equirecursive semantics cannot (without the undesirable possibility of potentially infinitely applying recursive definitions, in a so-called matching loop [8]). Nonetheless, an equirecursive semantics is much closer to an intuitive runtime model for a methodology, and theoretical papers which formalise verification logics typically treat recursive definitions in this way. This creates a mismatch between the formalised assertion logic semantics, and that typically implemented, which this paper addresses.

Implicit dynamic frames [15] extends a first-order logic with explicit assertions to describe permissions: an assertion $\operatorname{acc}(x . f)$ denotes permission to the heap location denoted by $x . f$. The key difference with separation logic is the inclusion of heap-dependent expressions in the logic; $x . f>0$ can be directly used as part of an assertion. To date, the logic has been used as the basis of three existing verifiers; Vericool [15], Chalice [7] and Syxc. While the symbolic execution engine underlying the first of these three verifiers has been proven sound [16], until recently no direct semantics for implicit dynamic frames assertions had been defined or studied. In recent work, Parkinson and Summers $[12,13]$ provide such an assertion logic semantics (and study its comparison with that of separation logic), including only the "core" of the assertion language, without any support for recursive definitions. Our paper also addresses this problem.

In this paper, we provide two formal semantics for an implicit dynamic frames logic including recursive functions and predicates; we give both isorecursive semantics (suitable as the basis for a verifier) and equirecursive semantics (suitable for comparison with a runtime model, and proving soundness). The development of such formalisations requires addressing several subtle issues, regarding both the possibility of infinitely-recursive definitions and the need for the isorecursive semantics to correctly reflect the restrictions that make it readily implementable. These questions are made more challenging still in the context of implicit dynamic frames, where the use of heap-dependent expressions (including recursively-defined functions) provides further pitfalls for a correct formal treatment. However, our formalisation can also be applied to other permissionbased logics, such as separation logic (in which only recursive predicates feature).

\footnotetext{
${ }^{3}$ Some tools provide these ghost operations with different names, or try to infer the location of these operations automatically, but the eventual meaning is the same.
} 
We discuss how our isorecursive model lends itself to implementation, and the related possibility of isorecursive states not having a "real" equirecursive counterpart. We define mappings from the isorecursive model to the equirecursive, and show how the various corresponding concepts are formally related. We extend both assertion semantics to corresponding Hoare Logics based on a subset of the Chalice programming language, and prove soundness with respect to an interleaving operational semantics.

\section{Equi-Recursive Semantics for Predicates and Functions}

In this section we define the syntax and semantics of expressions and assertions in the equi-recursive setting. Our treatment is based on the work of Parkinson and Summers [12]. Their work did not include any kind of predicates or functions in the assertion language; we address these issues here.

\subsection{Implicit Dynamic Frames}

Implicit dynamic frames allow expressions $e$ which depend on the heap, e.g., assertion $x . f . g=$ this. In order to make the meaning of such assertions robust to interference from other threads, a notion of permission is employed.Special assertions $\operatorname{acc}($ e.f) called accessibility predicates denote a permission to access the heap location e.f, at most one of which is present in the system at any one time. Assertions used in specifications should be self-framing, which means they must have permissions to all heap locations that they dereference in expressions. For example, the assertion $x . f . g=$ this is not self-framing, but $\operatorname{acc}(x . f) * \operatorname{acc}(x . f . g) * x . f . g=$ this is. The separating conjunction $*$ is related to that of separation logic; it acts just as logical conjunction, but behaves multiplicatively with respect to accessibility predicates; that is, $\operatorname{acc}(x . f) * \operatorname{acc}($ this. $f)$ requires permission to both locations separately, and so its meaning implicitly guarantees that this and $x$ cannot be aliases.

Defining a formal assertion semantics for implicit dynamic frames is challenging. Parkinson and Summers [12] defined a semantics for a core of the logic, and, among other questions, addressed the question of the semantics of assertions which are not self-framing. For example, what should be the meaning of $x . f . g=$ this ? In a state which does not hold permissions to the two heap locations, evaluation of this expression depends on the other threads, and so giving it a deterministic semantics seems incorrect. However, the difficulty is that a compositional definition of the semantics of assertions cannot "see" whether the appropriate permissions to $x . f$ and $x . f . g$ are held by the current thread. The solution used in [12] is, to give the expression the semantics it should have assuming the appropriate permissions are held; i.e., read from the heap regardless. Since all assertions are additionally checked to be self-framing, in the end this means that the above semantics is only applied in the situation in which it

makes sense. As we will show in subsection 2.3, similar issues arise when adding recursive definitions to the logic, but they cannot be resolved so simply. 


\subsection{Recursive Predicates and Functions}

Implicit dynamic frames supports two kinds of recursive definitions in assertions. In this paper, we use the terminology of the Chalice tool [7], and call them predicates and functions. Predicates can be defined recursively, and their bodies are assertions. Allowing specifications to mention predicates as well as field permissions and boolean expressions, makes it possible for, e.g., a precondition to require all permissions to a recursive data structure. For example, a predicate $P$ storing all permissions in a linked list could be defined by: $P \equiv \operatorname{acc}($ this.next $) * \operatorname{acc}($ this. $v a l) *($ next $\neq$ null $\rightarrow$ next.P $)$

Implicit dynamic frames also support recursively-defined functions as part of the expression syntax. For example, the assertions this.itemAt(2) $=4$ uses a function. Functions typically correspond to the "pure methods" of an implementation ${ }^{4}$. Function definitions have preconditions, which firstly, must require permissions to the heap locations accessed by the function's body, and secondly, they must guarantee termination of the body's evaluation.

Termination of function definitions is essential for soundness, once they are permitted in assertions. For example, a definition $f()=1+f()$ would allow the verifier to deduce inconsistency wherever this definition was made available.

\subsection{Handling infinite recursion}

The introduction of recursively-defined functions and predicates open the potential of non-termination. What should be the semantics of a predicate instance whose definition can be unrolled infinitely? And what should be done with function definitions that do not terminate? It is tempting to say that definitions which may not terminate should be forbidden. But this would be too restrictive: For example, even through the linked list predicate $P$ from the previous section does not terminate in a heap in which this $=$ this.next, such predicate definitions are essential for traversing recursive data structures, and cannot be dispensed with. Similarly, a function length ()$=($ this.next $=$ null $? 1: 1+$ next.length ()$)$ which takes an instance of predicate $P$ as pre-condition, does not terminate in the case that this = this.next. Indeed, there is not even any obvious termination measure that could be used to prove termination of this function definition, without assuming that the definition of $P$ itself terminates. Such functions cannot be dispensed with either.

The discussion above motivates making the semantics of infinitely-unrollable predicate instances false. In this way, we build into the logic the implicit assumption that all predicate instances have finite definitions. This enables a proof of the termination of function length() above, by assuming the termination of the predicate instance taken as precondition. Forbidding infinite predicate instances does not harm our expressiveness in practice, since, as will be explained in the next section, such predicate instances could never be obtained in a verifier based on an isorecursive semantics. Thus, any program point at which an infinite predicate instance is required (for example, in a method precondition of the form

\footnotetext{
${ }^{4}$ Indeed, this is the terminology used in [15].
} 
$P *$ this $=$ this.next) is actually unreachable code, and thus it is operationally consistent to assign false to such assertions.

Now consider the semantics of potentially-non-terminating functions. Since function definitions must be proved to terminate under the assumption that their preconditions hold (and that all predicate instances from their preconditions terminate themselves), it is sufficient to check that functions are only mentioned in assertions which also require their preconditions; we build this into the notion of an assertion being self-framing. However, this still doesn't yield a well-defined semantics for the function calls themselves. For example, consider the assertion $P *$ length ()$=3$. In the case where this $=$ this.next holds, $P$ will be false, and the overall assertion should also mean false. But a naïve definition for expression semantics might give the function call an ill-defined meaning. To avoid the need for relying on a short-cutting semantics for conjunctions, we define an expression semantics that is total, even for naturally non-terminating function calls. We achieve this by the introduction of error values, which are dummy values used in place of a non-terminating expression evaluation. Since the overall assertion will be false whenever these error values occur, this means that we implement the natural semantics for function calls in all situations where the meaning matters.

\subsection{Syntax}

Definition 1 (Expressions and Assertions). We define the syntaxes of equiexpressions (ranged over by $e$ ) and equi-assertions (ranged over by a), by the following grammars, in which $x$ ranges over program variables, $f$ ranges over field identifiers, $g$ over function names, $P$ over predicate names, and $q$ ranges over rational numbers in the range $(0,1]$ :

$$
\begin{aligned}
& e::=\text { null } \mid \text { true } \mid \text { false }|x| \text { e.f } \mid \text { e.g }(e)|e=e|(e ? e: e) \\
& a::=e|\operatorname{acc}(e . f, q)| e \rightarrow a|a * a| \text { e.P }
\end{aligned}
$$

We implicitly expect expressions and assertions to be type-correct, e.g. in e.f the type of $e$ should have a field $f$. Other connectives, such as $\wedge, \vee$ and $\neg$ can be encoded. To keep the presentation simple, we only support full permissions on predicates; i.e., we do not allow predicate instances themselves to be "split". Some tools support this, but an extension is straightforward. Note that the implication connective included in our assertion language is restricted to only boolean expressions on the left-hand side. This restriction is common to most practical verification tools based on separation logic or implicit dynamic frames; it makes it possible to avoid an assertion semantics which needs to quantify over states (see e.g., [9]); a problematic feature for automatic verification. Similarly, negation is only supported for boolean expressions. Thus, acc(this. $f, 1) *$ this. $f=$ 5 , and this.f $=5 \rightarrow \operatorname{acc}($ this. $f, 1)$ are assertions according to our definitions, while $\operatorname{acc}($ this. $f, 1) \rightarrow$ this. $f=5$ is not.

\subsection{Semantics}

As in [12], semantics of assertions is defined in terms of permission masks $\pi$, heaps $H$, and frames $\sigma$. As usual, we need a $*$ operator to combine permissions. 


\section{Definition 2 (Preliminaries).}

We assume a set of values consisting of null, true, object addresses (denoted by ८), and one distinct error value per type $t$ (denoted by error ${ }_{t}$ ).

We define equi-permission-masks $\pi \in$ perms, heaps $H \in H e a p$, environments $\sigma$, as mappings from variables to addresses, resp. object addresses and field identifiers to values, resp. from object address and field identifiers to values in [0..1].

We define the operator + to combine permissions as follows:

$$
+: \text { perms } \times \text { perms } \rightarrow \text { perms and }\left(\pi+\pi^{\prime}\right)(\iota, f)=\pi(\iota, f)+\pi^{\prime}(\iota, f) .
$$

Equi-permission-mask is well-defined, $=\pi$, if all values are between 0 and 1:

$$
\models \pi \quad \text { iff } \quad \forall \iota, f . \pi(\iota, f) \in[0 . .1]
$$

A lookup function Body returns the definition of a function, or a predicate respectively. For simplicity, predicates are parameterised by this, and functions have the implicit parameterised by this, and one further parameter, x. A lookup function Pre returns the precondition of a function.

A novelty in definition 2 is the error value, error $_{t}$, which we motivated earlier; it is used to define the value of expressions when their evaluation is infinite:

\section{Definition 3 (Value of Equi-Recursive Expressions).}

We define evaluation of expressions in a state consisting of heap $H$, and stack frame $\sigma$, through predicate $\Downarrow_{H, \sigma}$ - as follows:

$$
\begin{array}{lc}
x \Downarrow_{H, \sigma} \sigma(x) & \text { null } \Downarrow_{H, \sigma} \text { null true } \Downarrow_{H, \sigma} \text { true } \\
\text { e.f } \Downarrow_{H, \sigma} v & \text { if } e . f \Downarrow_{H, \sigma} \iota \text { and } H(\iota, f)=v . \\
\text { e.g }\left(e^{\prime}\right) \Downarrow_{H, \sigma} v & \text { if } e \Downarrow_{H, \sigma} \iota \text { and } e \Downarrow_{H, \sigma} v^{\prime} \text { and Body }(g) \Downarrow_{H, \sigma^{\prime}} v, \\
\text { where } \sigma^{\prime}=[\text { this, } x \mapsto \iota, v] . \\
e=e^{\prime} \Downarrow_{H, \sigma} \text { true } & \text { if } e \Downarrow_{H, \sigma} v \text { and } e \Downarrow_{H, \sigma} v \text { for some } v . \\
e=e^{\prime} \Downarrow_{H, \sigma} \text { false } & \text { if } e \Downarrow_{H, \sigma} v \text { and } e \Downarrow_{H, \sigma} v^{\prime} \text { and } v \neq v^{\prime} . \\
\left(e_{1} ? e_{2}: e_{3}\right) \Downarrow_{H, \sigma} v & \text { if } e_{1} \Downarrow_{H, \sigma} \text { true and } e_{2} \Downarrow_{H, \sigma} v . \\
\left(e_{1} ? e_{2}: e_{3}\right) \Downarrow_{H, \sigma} v & \text { if } e_{1} \Downarrow_{H, \sigma} \text { false and } e_{3} \Downarrow_{H, \sigma} v .
\end{array}
$$

We now define the value of an expression e in the context of $H$ and $\sigma$, as follows:

$$
\|e\|_{H, \sigma}=\left\{\begin{array}{lr}
v & \text { if } e \Downarrow_{H, \sigma} v \\
\text { error }_{t} & \text { if } \nexists v . e \Downarrow_{H, \sigma} v \text { and } \text { e has type } t .
\end{array}\right.
$$

Thus, in a configuration $H_{0}, \sigma_{0}$ where $z$ points to a cycle, $\| z$.length ()$\|_{H_{0}, \sigma_{0}}=$ error $_{\text {int }}$, and $\| z$.length $=3 \|_{H_{0}, \sigma_{0}}=$ error $_{\text {bool }}$. We can represent $z$.length $\neq 3$ through $(z$ length $=3$ ? false: true $)$, and obtain $\|(z$.length $=3$ ? false $:$ true $) \|_{H_{0}, \sigma_{0}}=$ error $_{\text {bool }}$. The presence of error values in the above definition may seem surprising, but we will shortly show how that they can only occur when the meaning of the expression is irrelevant to the assertion in which it occurs. First, we must define the semantics of equi-recursive assertions:

Definition 4 (Equi-Recursive Assertion Semantics). We define the semantics of assertions in a state comprising of permissions $\pi$, heaps $H$, and 
stack frame $\sigma$, as follows:

$$
\begin{array}{ll}
\pi, H, \sigma \models_{\mathrm{E}} e & \Longleftrightarrow\|e\|_{\pi, H, \sigma}=\text { true } \\
\pi, H, \sigma \models_{\mathrm{E}} \operatorname{acc}(e . f, q) & \Longleftrightarrow \pi\left(\|e\|_{\pi, H, \sigma}, f\right) \geq q \\
\pi, H, \sigma \models_{\mathrm{E}} e \rightarrow a & \Longleftrightarrow\|e\|_{\pi, H, \sigma} \Rightarrow \pi, H, \sigma \models_{\mathrm{E}} a \\
\pi, H, \sigma \models_{\mathrm{E}} a_{1} * a_{2} & \Longleftrightarrow \exists \pi_{1}, \pi_{2}: \pi=\pi_{1}+\pi_{2} \\
& \\
\pi, H, \sigma \models_{\mathrm{E}} e . P & \Longleftrightarrow \pi, \pi_{1}, H, \sigma \models_{\mathrm{E}} a_{1} \text { and } \pi_{2}, H \\
&
\end{array}
$$

Equi-entailment $a \models_{\mathrm{E}} a^{\prime}$ holds if: $\forall \pi, H, \sigma . \pi, H, \sigma \models_{\mathrm{E}} a \Longrightarrow \pi, H, \sigma \models_{\mathrm{E}} a^{\prime}$.

Thus, in $H 0, \sigma_{0}$ from above we have $\pi, H_{0}, \sigma_{0} \nvdash_{\mathrm{E}} z$.length $=3$, and $\pi, H_{0}, \sigma_{0} \not \nvdash_{\mathrm{E}}$ z.length?true : false. Note that $\pi, H, \sigma \models_{\mathrm{E}}$ e.P holds, if $\pi, H, \sigma \models_{\mathrm{E}} \operatorname{Body}(P)[e /$ this $]$ holds in a finite unfolding. Therefore, we also obtain $\pi, H_{0}, \sigma_{0} \not \nvdash_{\mathrm{E}} z$.Acyclic.

We can encode z.length $\neq 3$ also through z.length $=3 \rightarrow$ false, and have $\pi, H_{0}, \sigma_{0} \models_{\mathrm{E}}$ z.length $=3 \rightarrow$ false. This seems to be a concern given that $\pi, H_{0}, \sigma_{0} \not \nvdash_{\mathrm{E}}$ z.length?true : false. However, these concerns are eliminated once we add the definition of framed expressions and assertions. Essentially, we define a judgement which ensures that a particular state holds enough permissions to access all fields, and guarantee all preconditions of functions, on which the meaning of a particular expression/assertion depends. We then define an assertion to be self-framing if it can only be made true in states in which it is framed. In this way, we guarantee that all assertions are either trivially false, or else their semantics will not include calculation of error values, and thus the intuitive semantics of expressions is restored.

Definition 5 (Framed and Self-Framing equi-assertions). An equi-expression $e$, or assertion $a$ is equi-framed in a state consisting of $H, \pi$ and $\sigma$, under the following conditions:

$$
\begin{aligned}
& \models_{\text {frmE }}^{\pi, H, \sigma} \text { null } \quad \models_{\text {frmE }}^{\pi, H, \sigma} \text { true } \models_{\text {frmE }}^{\pi, H, \sigma} \text { false } \models_{\text {frmE }}^{\pi, H, \sigma} x
\end{aligned}
$$

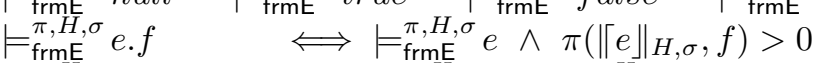

$$
\begin{aligned}
& \models_{\mathrm{frmE}}^{\pi, H, \sigma} \text { e.g }\left(e^{\prime}\right) \Longleftrightarrow \models_{\mathrm{frmE}}^{\pi, H, \sigma} \text { e } \wedge \models_{\mathrm{frmE}}^{\pi, H, \sigma} e^{\prime} \wedge \pi, H, \sigma^{\prime} \models_{\mathrm{I}} \operatorname{Pre}(g) \\
& \text { where } \sigma^{\prime}=\left[\text { this, } x \mapsto\|e\|_{H, \sigma},\left\|e^{\prime}\right\|_{H, \sigma}\right] \\
& \models_{\mathrm{frmE}}^{\pi, H, \sigma} e=\left.e^{\prime} \Longleftrightarrow\right|_{\mathrm{frmE}} ^{\pi, H, \sigma} e \wedge \models_{\mathrm{frmE}}^{\pi, H, \sigma} e^{\prime} \\
& \left.\right|_{\mathrm{frmE}} ^{\pi, H, \sigma} e ? e^{\prime}:\left.e^{\prime \prime} \Longleftrightarrow\right|_{\mathrm{frmE}} ^{\pi, H, \sigma} e \wedge\left(\pi, H,\left.\left.\sigma\right|_{\mathrm{I}} e \Rightarrow\right|_{\mathrm{frmE}} ^{\pi, H, \sigma} e^{\prime}\right)
\end{aligned}
$$

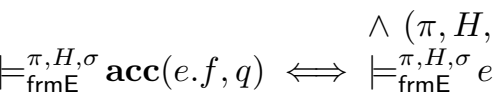

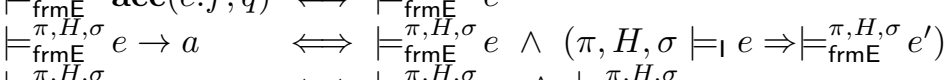

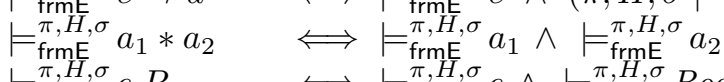

$$
\begin{aligned}
& ={ }_{\mathrm{frmE}}^{\pi, H, \sigma} e \cdot P \quad \Longleftrightarrow=_{\mathrm{frmE}}^{\pi, H, \sigma} e \wedge \|_{\mathrm{frmE}}^{\pi, H, \sigma} \operatorname{Body}(P)[e / \text { this }]
\end{aligned}
$$

The last case of the above definition may not give a well-defined true or false meaning to $\models_{\text {frmE }}^{\pi, H, \sigma}$ e.P; in this case, we make the meaning false by default.

An equi-assertion $a$ is self-framing, written $\models_{\text {frmE }} a$ if, for all $H, \pi$ and $\sigma$ : $\pi, H, \sigma \models_{\mathrm{E}} a \Rightarrow \models_{\mathrm{frmE}}^{\pi, H, \sigma} a$

Thus, $x=3$ is always framed, and thus self-framing trivially, while $x . n e x t \neq$ null is framed only when the state holds permission to the heap location x.next. 
Similarly, x.length() is framed only in configurations satisfying its precondition. Note that the notion of being framed does not unroll recursive definitions; we still need that these definitions are sensible. Therefore, we now combine this definition with a notion of well-definedness for function and predicate definitions, in order to guarantee that a framed assertion always has a meaningful semantics.

Definition 6 (Equi-well-formed predicate and function definitions). $A$ function $f$ 's definition (with body e of type $t$ ) is well-formed, if: (1) $\models_{\text {frmE }} \operatorname{Pre}(f)$, and (2) $\forall \pi, H, \sigma .\left(\pi, H, \sigma \models_{\mathrm{E}} \operatorname{Pre}(f) \Longrightarrow=_{\mathrm{frmE}}^{\pi, H, \sigma} e \wedge\|e\|_{H, \sigma} \neq\right.$ error $\left._{t}\right)$

A predicate $p$ 's definition is well-formed, if $\models_{\text {frmE }} \operatorname{Body}(p)$.

The condition $\|e\|_{H, \sigma} \neq$ error $_{t}$ for function definitions amounts to guaranteeing that the function evaluation terminates whenever the function pre-condition holds. Note that, because of our semantics of predicates, this proof may depend on the fact that any predicate instances required in the function's precondition have terminating definitions; otherwise the precondition would be false.

The definitions provided in this section give a direct semantics to well-defined recursive functions and predicates, in which function calls are always equal to their bodies (provided their preconditions hold), and predicates are also evaluated in terms of their bodies directly. Furthermore, the notions of framing and well-definedness for recursive definitions involve a recursive unrolling of the entire definitions. This is indeed the equi-recursive sense of the concepts, but is not suitable as the basis for an implementation. In the next section, we turn to the corresponding isorecursive notions.

\section{Iso-Recursive Semantics for Predicates and Functions}

In this section, we introduce an assertion semantics in the iso-recursive style. In the iso-recursive approach, predicates are differentiated from their bodies; this is handled in the logic by treating predicate names merely as another kind of permission, which can be rewritten into the corresponding body of the predicate by explicit extra fold and unfold statements in the program. With this approach, there are then two different notions of permission a thread can have; the explicit permissions, which can be represented in a permissions masks as usual, and the implicit permissions, which are those which are folded (perhaps recursively) inside of predicates held in the explicit permissions. To differentiate isorecursive definitions from their equirecursive counterparts, we typically use corresponding upper-case metavariables ( $\Pi$ for masks, $E$ for expressions, etc.).

Definition 7 (Iso-Expressions and Assertions). We define the syntaxes of iso-expressions (ranged over by E) and iso-assertions (ranged over by A), by the following grammars, in which $x$ ranges over program variables, $f$ ranges over field identifiers, $g$ over function names, $P$ over predicate identifiers, and $q$ ranges over rational numbers in the range $(0,1]$ :

$E::=$ null $\mid$ true $|x| E . f|E . g(E)| E=E|(E ? E: E)|$ unfolding $E . P$ in $E$ $A::=E|\operatorname{acc}(E . f, q)| E \rightarrow A|A * A| \operatorname{acc}(E . P)$ 
The difference between a permission to an abstract predicate, denoted $\operatorname{acc}(E . P)$ above, and the equi-recursive assertion $e . P$ will shortly be seen in the semantics. The syntax of iso-recursive expressions is similar to that of equi-recursive expressions. The only difference is the expression unfolding $E_{1} . P$ in $E_{2}$ which did not exist in the equi-recursive setting. The value of this expression is the same as that of $E_{2}$, however, the way such an expression is checked to be framed in a state is different; the unfolding of the predicate $P$ means that $E_{2}$ may depend on permissions which come from the body of $E_{1} . P$.

Definition 8 (Semantics of Iso-Recursive Expressions). We define the evaluation of iso-expressions $E$ in a state comprising of heaps $H$, and environment $\sigma$, in the analogous manner to definition 3, for example,

$$
E=E^{\prime} \Downarrow_{H, \sigma} \text { false if } E \Downarrow_{H, \sigma} v \text { and } E \Downarrow_{H, \sigma} v^{\prime} \text { and } v \neq v^{\prime} \text {. }
$$

with the following additional case:

unfolding $E_{1}$.P in $E_{2} \Downarrow_{H, \sigma} v \quad$ if $E_{2} \Downarrow_{H, \sigma} v$.

Moreover, as in definition 3 , if $E$ has type $t$, then

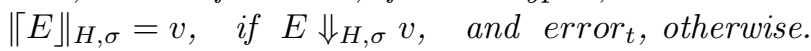

The full definition appears in the appendix.

In order to model iso-recursive assertions, we extend the concept of permission mask, so that it also holds permissions to predicate instances.

Definition 9 (Iso-Recursive Permissions). Iso-recursive permission masks, $\Pi \in$ Perms, are mappings from addresses and field identifiers, to non-negative values in $\mathbb{Q}$, and from addresses and predicate identifiers to non-negative values in $\mathbb{Z}$.

The function $\mathcal{P}$ collects the permissions explicitly required by an assertion, $\mathcal{P}:$ IsoAssertion $\times$ Heap $\times$ Env $\rightarrow$ Perms

$$
\begin{array}{lll}
\mathcal{P}(E, H, \sigma) & =\emptyset \\
\mathcal{P}(\operatorname{acc}(E . f, q), H, \sigma) & =\left\{\left(\|E\|_{H, \sigma}, f\right) \mapsto q\right\} \\
\mathcal{P}\left(E \rightarrow A^{\prime}, H, \sigma\right) & =\mathcal{P}(A, H, \sigma) \text { if }\|E\|_{H, \sigma}=\text { true, } \\
\mathcal{P}\left(A * A^{\prime}, H, \sigma\right) & \emptyset \text { otherwise } \\
\mathcal{P}(\operatorname{acc}(E . P), H, \sigma) & =\left\{\left(\|E\|_{H, \sigma}, f\right) \mapsto \mathcal{P}\left(A^{\prime}, H, \sigma\right)\right.
\end{array}
$$

The operation $\odot$, applied to permission mask $\Pi$, address $\iota$ and predicate identifier $P$, removes the permission to $\iota . P$ from the $\Pi$, and adds all the permissions obtained by unfolding the predicate body once.

$$
\Pi \odot_{H} \iota . P=\Pi[(\iota, P) \mapsto \Pi(\iota, P)-1]+\mathcal{P}(\operatorname{Body}(P), H, \sigma), \quad \text { where } \sigma(\text { this })=\iota .
$$

An interesting point in the definition above is the liberal treatment of permission masks $\Pi$, which allows permissions to fields be any rational numbers, even if they exceed 1. In the isorecursive setting, because we do not work with full knowledge of the permissions contained within predicate instances, we cannot in general rule out the possibility that the assertions we handle implicitly require more than the full permission to a field. In other words, there is always the possibility for isorecursive permission masks to have no corresponding well-formed equirecursive permission mask. For uniformity, therefore, we ignore this issue in our isorecursive model, and address it in the following section. 
Definition 10 (Semantics of Iso-Recursive Assertions). We define the semantics of iso-assertions in a state comprising of an iso-permission mask $\Pi$, heap $H$, and an environment $\sigma$, by the following:

$$
\begin{aligned}
& \Pi, H, \sigma \models_{1} E \quad \Longleftrightarrow\|E\|_{H, \sigma}=\text { true } \\
& \Pi, H, \sigma \models_{1} \operatorname{acc}(E . f, q) \Longleftrightarrow \Pi\left(\|E\|_{H, \sigma}, f\right) \geq q \\
& \Pi, H, \sigma \models_{1} E \rightarrow A \quad \Longleftrightarrow \Pi, H, \sigma \models_{1} E \Rightarrow \Pi, H, \sigma \models_{1} A \\
& \Pi, H, \sigma \models_{1} A_{1} * A_{2} \quad \Longleftrightarrow \exists \Pi_{1}, \Pi_{2}: \Pi=\Pi_{1}+\Pi_{2}, \Pi_{1}, H, \sigma \models_{1} A_{1}, \Pi_{2}, H, \sigma \models_{1} A_{2} \\
& \Pi, H, \sigma \models_{1} \operatorname{acc}(E . P, n) \Longleftrightarrow \Pi\left(\|E\|_{H, \sigma}, P\right) \geq n
\end{aligned}
$$

Iso-entailment $A \models_{\mathrm{E}} A^{\prime}$ holds if: $\forall \Pi, H, \sigma . \Pi, H, \sigma \models_{\mathrm{E}} A \Longrightarrow \Pi, H, \sigma \models_{\mathrm{E}} A^{\prime}$.

Crucially, the semantics of predicate permissions $\operatorname{acc}(E . P, n)$ do not involve recursion; it is sufficient to simply check that permission to the predicate instance is in the direct permissions. This does not directly enforce that the body of the predicate holds in the current state, but, as we shall show in the next section, we push this concern to the definition of a "good" isorecursive state; since a verifier cannot in general enforce that a recursive definition holds, this has to be pushed to the soundness of the underlying methodology (i.e., the equirecursive semantics).

We can now define the notion of framing for isorecursive expressions and assertions (cf. Definition 5).

Definition 11 (Framed and Self-Framing iso-assertions). An iso-assertion expression $E$, or assertion $A$ is iso-framed in a state consisting of $H, \Pi$ and $\sigma$, as defined by judgements $\models_{\mathrm{frml}}^{\Pi, H, \sigma} E$ and $\models_{\mathrm{frml}}^{\Pi, H, \sigma}$ A. Full definitions are provided in the appendix, but all cases of these judgements are analogous with those of Definition 5, with the following exceptions:

$$
\begin{aligned}
& \models_{\text {frml }}^{\Pi, H, \sigma} \text { unfolding } E . P \text { in } E^{\prime} \Longleftrightarrow \models_{\text {frml }}^{\Pi, H, \sigma} E \wedge \Pi\left(\|E\|_{H, \sigma}, P\right) \geq 1 \wedge \models_{\text {frml }}^{\Pi^{\prime}, H, \sigma} E^{\prime} \text {, } \\
& \models_{\text {frml }}^{\Pi, H, \sigma} \operatorname{acc}(E . P, q) \quad \Longleftrightarrow \models_{\text {frml }}^{\Pi, H, \sigma} E \\
& \text { An iso-assertion } A \text { is self-framing, written }=_{\mathrm{frml}} A \text { if, for all } H, \Pi \text { and } \sigma \text { : } \\
& \Pi, H, \sigma \models_{\mathrm{l}} A \Rightarrow \models_{\text {frml }}^{\Pi, H, \sigma} A
\end{aligned}
$$

Most importantly, the notion of framing no longer requires a recursive traversal of predicate definitions. This can only be justified with two further ingredients; firstly, that predicate definitions are always self-framing, and secondly that holding a predicate always implicitly guarantees that its body holds in the same state. The former of these two ingredients is provided by the following definition, while the second is provided by the notion of "good state" in the next section.

Definition 12 (Iso-well-formed predicate and function definitions). $A$ function $f$ 's definition (with body $E$ of type $t$ ) is iso-well-formed, if: (1) $\models_{\text {frml }}$ $\operatorname{Pre}(f)$, and $(2) \forall \Pi, H, \sigma .\left(\Pi, H, \sigma \models_{\mathrm{I}} \operatorname{Pre}(f) \Longrightarrow \models_{\mathrm{frml}}^{\Pi, H, \sigma} \operatorname{Body}(f) \wedge \mid\left[E \|_{H, \sigma} \neq\right.\right.$ error $_{t}$ )

A predicate $p$ 's definition is iso-well-formed, if $\models_{\text {frml }} \operatorname{Body}(p)$.

A program is well-formed if all of the function and predicate definitions are well-formed. 
Note that this notion of well-formedness, unlike that of Definition 12 can be checked without unrolling definitions recursively. In the next section, we will show how the isorecursive definitions above correctly approximate the corresponding equirecursive notions.

\section{Comparing the Assertion Semantics}

We now turn to relating our two semantics. Our eventual goal is to define an erasure, mapping our isorecursive constructions to their equirecursive counterparts, in order to show that verification based on isorecursive semantics gives a sound approximation of verification based on equirecursive semantics. In particular, we will show that fold, unfold and unfolding (which are essential for defining semantics in the isorecursive sense) can all be eliminated from the language, and the resulting program still satisfies the erased version of its specifications. Since permissions (let alone permissions to predicates) are not reflected at runtime, this leads us closer to a runtime model suitable for proving soundness with respect to an operational semantics. In this section, we focus on the relationship between the two semantics for assertions.

Definition 13 (Encoding). The encoding $\langle\langle-\rangle$ maps isorecursive expressions and assertions to their equirecursive counterparts, typically by injection, e.g.

$$
\langle\langle x\rangle\rangle=x, \quad\langle\langle E . f\rangle\rangle=\langle\langle E\rangle . f, \quad \text { and } \quad\langle\langle\operatorname{acc}(E . f, q)\rangle=\operatorname{acc}(E . f, q) .
$$

For the cases specific to iso-expressions and assertions, we have

$\left\langle\left\langle\right.\right.$ unfolding $E . P$ in $\left.\left.E^{\prime}\right\rangle\right\rangle=\left\langle\left\langle E^{\prime}\right\rangle\right\rangle, \quad$ and $\quad\langle\langle\operatorname{acc}(E . P)\rangle=\langle\langle E\rangle\rangle . P$. The full definition appears in the appendix.

Unfolding expressions are unnecessary in the equirecursive world, where predicates and their bodies are not differentiated between. However, the unrolling of predicate definitions may still lead to the discovery that too many field permissions were implicitly held in an isorecursive state; not all isorecursive masks have an equirecursive analogue. This is described in the next definition.

Definition 14 (Encoding permissions). The (partial) translation $\langle\langle-\rangle\rangle$ encodes isorecursive permission maps $\Pi$ into equirecursive permission maps $\pi$,

$$
\begin{aligned}
\langle\langle\Pi\rangle\rangle_{H} & =\{(\iota, f) \mapsto q \mid \Pi[\iota, f]=q\}+\left\langle\left\langle\Pi^{\prime}\right\rangle\right\rangle_{H} \\
\text { where } \Pi^{\prime} & =\sum_{\Pi(\iota . P)>0}(\mathcal{P}(\operatorname{Body}(P), H,[\text { this } \mapsto \iota]))
\end{aligned}
$$

Note that $\langle\langle\Pi\rangle\rangle_{H}$ may not be well-defined (if a predicate instance held in $\Pi$ has an infinite unfolding, then the definition above will not terminate), and even when defined, it may not yield a well-defined equi-permissions-mask (cf. Definition 2), if too many field permissions are accumulated. Furthermore, it could be the constraints required in the bodies of predicates are not always correctly reflected in an iso-recursive state. Thus, we define the notion of a "good" isorecursive state.

Definition 15 (Good Iso-States). An isorecursive state defined by heap $H$, iso-permissions-mask $\Pi$ and environment $\sigma$ is "good", if: 
1. $\langle\langle\Pi\rangle\rangle_{H}$ is defined, and satisfies $\models\left(\langle\langle\Pi\rangle\rangle_{H}\right)$.

2. For all $\iota, P$ such that $\Pi[\iota, P]>0,\left(\left\langle\langle\Pi\rangle_{H}\right), H, \sigma^{\prime} \models_{\mathrm{E}} w . P\right.$ is satisfied, where $w$ is a fresh variable, and $\sigma^{\prime}$ is the environment $\sigma$ extended with the mapping $[w \mapsto \iota]$.

Note, in particular, that the second of these two requirements enforces that the original state does not hold permission to any predicate instance whose definition can be unrolled infinitely. As motivated in Section 2, such predicates are interpreted as false in the equirecursive semantics in any case, so ruling out such states in advance is consistent with this view. In general good iso-states are those which can have a meaningful corresponding equirecursive counterpart; all others are artifacts of the incomplete knowledge provided by isorecursive definitions.

We prove Lemma 1 which shows that, we can map a judgement back from the equirecursive world to the isorecursive, starting from a "good" state in the isorecursive world, and then, in Theorem 1, we show our erasure results.

Lemma 1. In a well-defined program,

1. If $\Pi(\iota . P)>0$, then $\langle\langle\Pi\rangle\rangle_{H}=\left\langle\left\langle\Pi \odot_{H} \iota . P\right\rangle\right\rangle_{H}$.

2. If $\pi, H, \sigma=_{\mathrm{E}}\left\langle\langle A\rangle\right.$, then $\exists \Pi$ s.t. $(\Pi, H, \sigma)$ is a good iso-state, $\left\langle\langle\Pi\rangle_{H}=\pi\right.$, and $\pi, H, \sigma \models$ $A$.

\section{Theorem 1 (Erasure Results).}

1. $\mid\left[\left\langle\langle E\rangle \|_{H, \sigma}=\right|\left\lceil E \|_{H, \sigma}\right.\right.$.

2. If $\Pi, H, \sigma \models_{\mathrm{I}} A$, and $(\Pi, H, \sigma)$ is a good iso-state, then $\left\langle\langle\Pi\rangle_{H}, H, \sigma \models_{\mathrm{E}}\right.$ $\langle\langle A\rangle$.

3. If $(\Pi, H, \sigma)$ is a good iso-state, and $\models_{\mathrm{frml}}^{\Pi, H, \sigma} A$, then $\models_{\mathrm{frmE}}^{\langle\langle\Pi\rangle, H, \sigma}\langle\langle A\rangle\rangle$.

4. If all functions and predicates are well-formed, then if $A$ is iso-self-framing, then $\langle\langle A\rangle$ is equi-self-framing

5. If $A=_{1} A^{\prime}$, then $\langle\langle A\rangle\rangle \models_{\mathrm{E}}\left\langle\left\langle A^{\prime}\right\rangle\right\rangle$.

The proof sketches are given in the appendix. These results demonstrate that the more-readily-checkable isorecursive notions supplied in the previous section, accurately approximate the intended underlying equirecursive notions. In the following sections, we extend this argument to Hoare Logics for a small language. We will then be in a position to prove that verifying a program with respect to isorecursive definitions, is sufficient to guarantee soundness, via our erasure results, and a soundness proof with respect to equirecursive semantics.

\section{Hoare Logic for the Iso assertions and programs}

In this section, we define an axiomatic semantics for a small (but representative) subset of Chalice, with respect to our iso-recursive assertion semantics (as defined in Section 3). This is the semantics closest to most implementations, but it is not so intuitive or useful as a runtime model. In the following section, we will define a corresponding Hoare Logic for our equirecursive semantics, and demonstrate the relationship between the two. 


\subsection{Chalice Syntax}

We begin by defining our Chalice subset:

Definition 16 (Isorecursive Chalice Syntax). We assume a set of predefined methods, ranged over by $m$, and functions, ranged over by $f$. For simplicity, methods are assumed to have exactly one parameter, and to always return a value. Furthermore, method names are assumed to be unique in the whole program.

Simple statements, ranged over by $R$, and statements, ranged over by $S$, are mutually defined by the following grammars:

$$
\begin{aligned}
R & ::=\operatorname{skip}|x:=E| x . f:=y \mid \text { return } x \mid x:=\text { new } c \mid\left(\text { if } B \text { then } S_{1} \text { else } S_{2}\right) \\
& || x:=\text { fork } y \cdot m(z) \mid y:=\text { join } x \mid \text { fold } x . p \mid \text { unfold } x . p \\
S & ::=R \mid(R ; S)
\end{aligned}
$$

A statement $S$ is return-ended if the right-most simple statement occurring in its structure is of the form return $x$; i.e., it can be constructed by the following sub-grammar: $S::=$ return $x \mid(R ; S)$

Composition of statements $s_{1}$ and $s_{2}$, for which we use the (overloaded) notation $\left(S_{1} ; S_{2}\right)$, results in a statement which represents appending the two sequences of simple statements; i.e., when $S_{1}$ is not a simple statement (say, $\left.S_{1}=\left(R ; S^{\prime}\right)\right)$, is defined by recursively rewriting $\left(\left(R ; S^{\prime}\right) ; S_{2}\right)=\left(R ;\left(S^{\prime} ; S_{2}\right)\right)$.

Our syntax only allows for sequential compositions to be nested to the right, which simplifies the definition of the operational semantics (see Section 7), since we do not need a separate concept of evaluation contexts for such a simple language. Our language only allows general expressions $e$ within variable assignments, and otherwise employs only program variables for expressions, but the generalisation is easily encoded (or made). Multi-threading is achieved by the ability to fork and join threads. The statement $w:=$ fork $m . y(z)$ has the meaning of starting an invocation of a call to method $m$ (with receiver $y$ and parameter $z$ ) in a new thread. The returned value (stored in $w$ ) is a token, which gives a means of referring to this newly-spawned thread. Such a token can be used to join the thread later (which has the operational meaning of waiting for the thread to terminate, and then receiving its return value); this is provided by the $x:=$ join $w$ statement. In order to model information about active thread$\mathrm{s} /$ tokens, we need to extend our assertion logic with an additional assertion Thread $(x, m, y, z)$, which has the meaning that $x$ stores a token corresponding to a forked thread executing method $m$ with receiver $y$ and parameter $z$. In order to represent this in our assertion semantics, we extend our heaps with extra thread objects, which store the information $m, y, z$ in ghost fields. These fields cannot be referred to directly in assertions, but we extend our semantic judgement with the following rule:

$$
\begin{array}{r}
\pi, H, \sigma=_{\mathrm{E}} \text { Thread }(x, m, y, z) \Leftrightarrow \pi[x, \text { recv }]=\pi[x, \text { param }]=\pi[x, \text { meth }]=1 \\
\wedge H[x . r e c v]=\sigma(y) \wedge H[x . \text { param }]=\sigma(z) \wedge H[\text { x.meth }]=m
\end{array}
$$

and identically for its isorecursive semantics. Thus, Thread $(x, m, y, z)$ is a selfframing assertion, which can be passed between threads to indicate which thread has permission to join a token. 
We do not include loops, since they present no relevant challenges compared with recursion. While we do not support a method call statement, we do allow the fork and join of method invocations. A normal method call of the form $x:=m . y(z)$ can be encoded by a sequence $(w:=$ fork $m . y(z) ; x:=$ join $w)$ (for some fresh variable $w$ ).

\subsection{Hoare Logic}

We now define the Hoare Logic corresponding to isorecursive assertion semantics.

Definition 17 (Isorecursive Hoare Logic). Isorecursive Hoare Triples are written $\vdash_{I}\{A\} S\left\{A^{\prime}\right\}$, where $A$ and $A^{\prime}$ are self-framing isorecursive assertions, and $S$ is an Isorecursive Chalice statement. The rules are shown in Figure 1. We leave implicit the requirement that $A$ and $A^{\prime}$ are always self-framing; in particular, whenever we write a triple (even as a new conclusion of a derivation rule), this requirement must be satisfied in addition to the explicit premises.

Our Hoare triples employ isorecursive assertions as pre- and post-conditions, with the restriction that the assertions used must always be self-framing. The restriction to self-framing assertions is important for soundness. For example, without this requirement, it would naturally be possible to derive triples such as $\vdash_{I}\{x . f=1\}$ (skip; skip) $\{x . f=1\}$, which, when evaluated at runtime, might not be sound (another thread could modify the location $x . f$ during execution). Indeed, in our soundness proof, the requirement that every thread has a selfframing pre-condition is essential to the argument.

Some of the rules (such as the treatment of conditionals, and the rule of consequence, $($ consI $))$ ) are standard, but others warrant discussion. The frame rule (frameI)) (whose role is to preserve parts of the state which are not relevant for the execution of the particular statement) is similar to that typically employed in separation logics [5]. The extra assertion $A^{\prime \prime}$ can be "framed on" under two conditions; firstly, that no variables mentioned in $A^{\prime \prime}$ are modified by the statement $s$, and secondly, that $A^{\prime \prime}$ is self-framing. The two conditions are necessary for similar reasons; if the execution of $s$ could change the meaning of $A^{\prime \prime}$, then to simplify conjoin it unchanged to both pre- and post-condition would be incorrect. The two ways in which the state can change in our language are through variable assignments (whose effects are tamed by the first requirement) and field assignments (which cannot affect the meaning of $A^{\prime \prime}$, since $A^{\prime \prime}$ is self-framing, and therefore comes along with sufficient permission to rule out assignment to the fields on which its meaning depends).

The (varassI) rule is similar to a standard Hoare Logic rule for assignment, but with the extra requirement that the expression to be assigned is readable in the pre-condition state. The condition $A=_{1} \operatorname{Readable}(E)$ is a shorthand for $\Pi, H, \sigma \models \mathrm{I} A$ implies that $\models_{\text {frml }}^{\Pi, H, \sigma} E$, and guarantees that fields are only read when appropriate permissions are known to be available, and functions are only applied when their pre-conditions are known in the state. The rule (fldassI) is slightly subtle: it must avoid the possibility of outdated heap-dependent expressions surviving the assignment; the requirement for full permission to the 


$$
\begin{aligned}
& \left.\overline{\vdash_{I}\{A\} \operatorname{skip}\{A\}}(\text { skip } I) \frac{A[E / x] \models \text { Readable }(E)}{\vdash_{I}\{A[E / x]\} x:=E\{A\}} \text { (varass } I\right) \\
& \overline{\vdash_{I}\{x \neq n u l l * \operatorname{acc}(x . f, 1)\} x . f:=y\{\operatorname{acc}(x . f, 1) * x . f=y\}}(\text { fldassI) } \\
& \overline{\vdash_{I}\{A\} \text { return } x\{A * \text { result }=x\}}(\text { ret } I)
\end{aligned}
$$

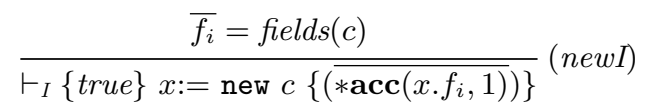

$$
\begin{aligned}
& \frac{\vdash_{I}\{A * B\} S_{1}\left\{A^{\prime}\right\} \quad \vdash_{I}\{A * \neg B\} S_{2}\left\{A^{\prime}\right\}}{\vdash_{I}\{A\}\left(\text { if } B \text { then } S_{1} \text { else } S_{2}\right)\left\{A^{\prime}\right\}} \text { (ifI) } \\
& \frac{A=\operatorname{Pre}(m)[y / \text { this }][z / \mathrm{x}]}{\vdash_{I}\{y \neq \operatorname{null} * A\} x:=\text { fork } y \cdot m(z)\{\operatorname{Thread}(x, m, y, z)\}}(\text { fork } I) \\
& \frac{A^{\prime}=\operatorname{Post}(m)[y / \text { this }][z / \mathrm{x}][w / \text { result }]}{\vdash_{I}\{\operatorname{Thread}(x, m, y, z)\} w:=\text { join } x\left\{A^{\prime}\right\}}(\text { join } I) \\
& \frac{\vdash_{I}\{A\} R\left\{A^{\prime}\right\} \quad \vdash_{I}\left\{A^{\prime}\right\} S\left\{A^{\prime \prime}\right\}}{\vdash_{I}\{A\}(R ; S)\left\{A^{\prime \prime}\right\}}(\text { seqI }) \\
& \left.\frac{A_{1} \models_{1} A_{3} \quad \vdash_{I}\left\{A_{3}\right\} s\left\{A_{4}\right\} \quad A_{4} \models_{1} A_{2}}{\vdash_{I}\left\{A_{1}\right\} s\left\{A_{2}\right\}} \text { (cons } I\right) \\
& \frac{\vdash_{I}\{A\} S\left\{A^{\prime}\right\} \quad A^{\prime \prime} \text { s.f. } \quad \operatorname{mods}(S) \cap F V\left(A^{\prime \prime}\right)=\emptyset}{\vdash_{I}\left\{A^{\prime \prime} * A\right\} S\left\{A^{\prime} * A^{\prime \prime}\right\}}(\text { frameI }) \\
& \frac{A^{\prime}=\operatorname{Body}(P)[x / \text { this }]}{\vdash_{I}\left\{A * A^{\prime} * B\right\} \text { fold } \operatorname{acc}(x . P, q)\{A * \operatorname{acc}(x . P, q) * \text { unfolding } x . P \operatorname{in} B\}}(\text { fold } I) \\
& \left.\frac{A^{\prime}=\operatorname{Bod} y(P)[x / \text { this }]}{\vdash_{I}\{A * \operatorname{acc}(x . P, q) * \text { unfolding } x . P \text { in } B\} \text { unfold acc }(x . P, q)\left\{A * A^{\prime} * B\right\}} \text { (unfold } I\right)
\end{aligned}
$$

Fig. 1. Hoare Logic for Isorecursive semantics

written field location from the pre-condition forces any information previouslyknown about that location to be discarded (i.e., using rule (consI)) prior to applying this rule.

The rules for forking and joining threads employ the special Thread $(x, m, y, z)$ assertion, discussed above. Our formulation does not allow this knowledge to be split amongst threads (though it can be passed from thread to thread in contracts). 
The two most important rules for the isorecursive semantics are those for folding and unfolding predicate instances. For example, consider folding a predicate instance, as defined by rule $($ fold $I)$ ). It is easy to see that this rule should exchange the body of the predicate instance (the assertion $A^{\prime}$ for a permission to the predicate itself). The challenge is to enable the preservation of information which was previously framed by the predicate's contents, even though those permissions have (after folding) been stored into the predicate body. For example, consider a predicate $P$ whose definition is $\operatorname{acc}($ this.g, 1$)$. In a state in which we know $\operatorname{acc}($ this.g) $*$ this.g $=4$, we could not treat a fold of $P$ as a simple exchange of $\mathbf{a c c}($ this.g) for $\operatorname{acc}($ this.P), since, in the resulting state, this.g $=4$ would not be framed. Instead, our rule allows us to derive the post-condition $\operatorname{acc}($ this.P) $*$ unfolding this.P in this.g $=4$, which is selfframing. Furthermore, in order to handle the possibility that we wish to preserve an expression which is framed partially by the permissions required by a predicate body, we allow the presence of a further assertion $A$ in the rule. This allows, e.g., a pre-condition such as $\operatorname{acc}($ this.f $) * \operatorname{acc}($ this.g) $*$ this.f $=$ this.g for a statement fold acc(this.P) to be used to derive a post-condition $\operatorname{acc}($ this.f $) * \operatorname{acc}($ this. $P) *$ unfolding this.P in this.f $=$ this.g, in which the additional assertion (acc(this.f) in this case) provides additional permissions required for self-framing. The condition that $A$ must be self-framing is necessary to avoid that $A$ itself might represent information which was only framed by permissions from the body of $P$.

The rule for unfolding predicates is exactly symmetrical with that for folding predicates. In particular, it enables information from unfolding expressions depending on the predicate to be unfolded, to be preserved (without an unfolding expression) in the post-state.

We can characterise the derivable triples in our Hoare Logic, using a generation lemma; an example case is shown here.

Lemma 2 (Generation Lemma).

$$
\begin{aligned}
& -\vdash_{I}\{A\} x:=E\left\{A^{\prime}\right\} \Leftrightarrow \\
& \quad \exists A^{\prime \prime} .\left(A^{\prime \prime} \text { s.f. } \wedge A \models_{1} A^{\prime \prime}[E / x] \wedge A^{\prime \prime}[E / x] \models_{I} \operatorname{Readable}(E) \wedge A^{\prime \prime} \models_{\mathrm{I}} A^{\prime}\right) \\
& \text { - Remaining cases in the appendix. }
\end{aligned}
$$

\section{Hoare Logic for the Equi assertions and programs}

In this section, we employ a second Hoare Logic based on our equirecursive assertion semantics. Firstly, we define an "erased" form of our statement syntax, in which only equi-recursive expressions are used, and no fold and unfold statements may occur.

Definition 18 (Equirecursive Chalice Syntax). Simple runtime statements, ranged over by $r$, and runtime statements, ranged over by s, are mutually defined by the following grammars:

$$
\begin{aligned}
r & ::=\operatorname{skip}|x:=e| x . f:=y \mid \text { return } x \mid x:=\text { new } c \mid\left(\text { if } b \text { then } s_{1} \text { else } s_{2}\right) \\
& \mid x:=\text { fork } y . m(z) \mid y:=\text { join } x \\
s & ::=r \mid(r ; s)
\end{aligned}
$$


The notions of return-ended statements and composition of statements are analogous to those of Definition 16.

We can now define the equirecursive analogue of Definition 17.

Definition 19 (Equirecursive Hoare Logic). Equirecursive Hoare Triples are written $\vdash_{E}\{a\} s\left\{a^{\prime}\right\}$, where $a$ and $a^{\prime}$ are self-framing equirecursive assertions, and $s$ is an equirecursive Chalice statement. For space reasons, the full rules are not given here. However, all rules are analogous to those of our Isorecursive Hoare Logic (Definition 17), except that the corresponding equirecursive notions of entailment, self-framing, statements, assertions etc. are used throughout. In addition, there are no rules for fold and unfold statements (since these do not occur in equirecursive Chalice). The full rules are given in the appendix (Figure 4).

We now extend our previous erasure results (mapping isorecursive to equirecursive assertions) to also define an erasure on statements. This operation applies erasure to all assertions and expressions, and replaces all fold/un fold statements with skip.

Definition 20 (Encoding iso-statements to equi-statements). We overload the encoding $\langle\langle-\rangle$ to map isorecursive to equirecursive statements, as:

$$
\begin{gathered}
\left\langle\langle x:=E\rangle=x:=\langle\langle E\rangle\rangle \quad\left\langle\left\langle\left(S_{1} ; S_{2}\right)\right\rangle\right\rangle=\left(\left\langle\left\langle S_{1}\right\rangle\right\rangle ;\left\langle\left\langle S_{2}\right\rangle\right\rangle\right)\right. \\
\left\langle\left\langle\left(\text { if } E \text { then } S_{1} \text { else } S_{2}\right)\right\rangle\right\rangle=\left(\text { if }\langle\langle E\rangle\rangle \text { then }\left\langle\left\langle S_{1}\right\rangle\right\rangle \text { else }\left\langle\left\langle S_{2}\right\rangle\right\rangle\right) \\
\langle\langle\text { fold } x . P\rangle\rangle=\operatorname{skip}=\langle\langle\text { unfold } x . P\rangle\rangle \\
\langle\langle S\rangle\rangle=S \text { otherwise }
\end{gathered}
$$

Theorem 2. If $A, A^{\prime}$ are self-framing iso-assertions, and $S$ is an isorecursive Chalice statement, then

$$
\left.\vdash_{I}\{A\} S\left\{A^{\prime}\right\} \Rightarrow \vdash_{E}\{\langle\langle A\rangle\rangle\}\langle\langle S\rangle\rangle\left\{\left\langle A^{\prime}\right\rangle\right\rangle\right\}
$$

We should show the proof for fold/unfold rules, at least.

\section{Soundness}

In this section, we show soundness of our formalisations, with respect to an interleaving small-step operational semantics. We formalise our runtime model with respect to a collection of threads and objects, together referred to as runtime entities. We do not model explicit object allocation; instead, we assume that all objects are pre-existing (and already have classes), but have a flag which indicates whether they are truly allocated or not. When unallocated, an object holds the permission to all of its own fields. Thus, we never need to create or destroy permission in the system; it is merely transferred from entity to entity. Similarly, we do not model creation of new threads, but just assume that idle thread entities exist in the system, which can be assigned a task (i.e., a method invocation) to begin executing. 
Definition 21 (Runtime Ingredients). We assume a set of object identifiers, ranged over by $\iota$, and a (disjoint) set of thread identifiers, ranged over by $t$. We assume a fixed mapping cls( $\iota)$ from object identifiers to class names.

$A$ thread configuration $T$ is defined by $T::=\{\sigma, s\} \mid$ idle

$A$ thread entity is a thread configuration labelled with a thread identifier, $T_{t}$.

An object state $O$ is defined by $O::=$ alloc $\mid$ free, and an object entity is an object state labelled with an object identifier, written $O_{\iota}$.

$A$ labelled entity $N_{n}$ is defined by $N_{n}::=T_{t} \mid O_{\iota}$, where the label $n$ denotes the thread or object identifier of the entity, respectively.

We define two main types of small-step transitions, which we call local and paired transitions. A local transition is one which affects only a single (thread) entity and the heap.

Definition 22 (Local transitions). Local transitions map a heap and thread entity to a new heap and thread entity (with the same thread identifier), and are written $h, T_{t} \rightarrow h^{\prime}, T^{\prime}{ }_{t}$. These rules have the expected shape, e.g.

$$
\frac{\|e\|_{h, \sigma}=v}{h,\{\sigma,(x:=e ; s)\}_{t} \longrightarrow h,\{\sigma[x \mapsto v], s\}_{t}}(\text { varass } S)
$$

The full rules for local transitions and are defined in Figure 5 in the appendix.

The more complex transitions are concerned with forking and joining threads, and with object allocation. In the case of forking and joining, we define transitions which simultaneously involve two thread entities; one which is executing the fork/join statement, and one which represents the thread being forked/joined. In the case of a fork, the second thread entity must be initially idle, while in the case of a join, it must have finished executing and be ready to return. Object allocation, on the other hand, takes an object entity in the free state (and of the correct class), and switches it to alloc.

Definition 23 (Paired transitions). Paired transitions map a heap and a pair of entities to a new heap and pair of entities (with the same identifiers), and are written $h,\left(T_{t} \| N_{n}\right) \longrightarrow h^{\prime},\left(T^{\prime}{ }_{t} \| N_{n}^{\prime}\right)$. They are defined in Figure 2.

We can now define the operational semantics for a whole system.

Definition 24 (Runtime Configurations and Operational Semantics). $A$ runtime configuration $C$ is a pair $\left(\overline{T_{t}}, \overline{O_{\iota}}\right)$ consisting of a set of thread entities (one for each thread identifier $t$ ) and a set of object entities (one for each object identifier $\iota$ ). The interleaving operational semantics of such a configuration is given by a transition relation of the form $C \underset{c}{\longrightarrow} C^{\prime}$, and is defined in Figure 3.

Definition 25 (Valid configuration). Let $C$ be a configuration, and let $S$ be the set of all active threads (i.e., all thread entities of the form $\{\sigma, s\}_{t}$, for some $\sigma, s, t)$ in $C$. The configuration $C$ is valid if there exists a set of equirecursive assertions $\overline{a_{t}}$, one for each thread entity $\left\{\sigma^{t}, s^{t}\right\}_{t}$ in $S$, such that $\models \mathcal{P}\left(*\left(\overline{a_{t}}\right)\right)$ and, for each, we have both $h, C, \sigma \models_{\mathrm{E}} a_{t}$ and $\vdash_{E}\left\{a_{t}\right\} s_{t}\left\{\operatorname{Post}\left(\sigma_{t}(\right.\right.$ method $\left.\left.)\right)\right\}$. 


$$
\begin{gathered}
\frac{\sigma(y)=\iota \quad \sigma^{\prime}=[\text { this } \mapsto \iota, \mathrm{x} \mapsto \sigma(z), \text { method } \mapsto m] \quad s^{\prime}=\operatorname{Body}(m)}{h,\left(\{\sigma,(x:=\text { fork } y . m(z) ; s)\}_{t_{1}} \| \text { idle }_{t_{2}}\right)}(\text { fork } S) \\
\frac{\sigma_{1}(y)=t_{2}}{p h,\left(\left\{\sigma\left[x \mapsto t_{2}\right], s\right\}_{t_{1}} \|\left\{\sigma^{\prime}, s^{\prime}\right\}_{t_{2}}\right)} \\
\frac{h_{p} h,\left(\left\{\sigma_{1}\left[x \mapsto \sigma_{2}(z)\right], s\right\}_{t_{1}} \| \text { idle } t_{2}\right)}{h,\left(\left\{\sigma_{1},(x:=\text { join } y ; s)\right\}_{t_{2}} \|\left\{\sigma_{2}, \operatorname{return} z t_{t_{2}}\right)\right.}(\text { join } S) \\
\frac{h^{\prime}=h \overline{\left[\left(\iota, f_{i}\right) \mapsto n u l l\right]}}{h,\left(\{\sigma,(x:=\text { new } c ; s)\}_{t} \| \text { free }(c)_{\iota}\right) \bar{p} h^{\prime},\left(\{\sigma[x \mapsto \iota], s\}_{t} \| \operatorname{alloc}(c)_{\iota}\right)}(\text { new } S)
\end{gathered}
$$

Fig. 2. Paired transitions

$$
\begin{gathered}
\frac{C[t]=\left(T_{t}\right) \quad h, T_{t} \underset{l}{l} h^{\prime}, T^{\prime}{ }_{t}}{h, C \underset{c}{c} h^{\prime}, C\left[t \mapsto T^{\prime}{ }_{t}\right]}(\text { selectLocal } S) \\
\left.\frac{C[t]=\left(T_{t}\right) \quad C[n]=\left(N_{n}\right) \quad h,\left(T_{t} \| N_{n}\right) \underset{p}{\longrightarrow} h^{\prime},\left(T^{\prime}{ }_{t} \| N^{\prime}{ }_{n}\right)}{h, C \underset{c}{\longrightarrow} h^{\prime}, C\left[t \mapsto T^{\prime}{ }_{t}\right]\left[n \mapsto N^{\prime}{ }_{n}\right]} \text { (selectPair } S\right)
\end{gathered}
$$

Fig. 3. Configuration transitions

Theorem 3 (Soundness of Verification). For a well-formed program, if all method definitions satisfy $\vdash_{I}\{\operatorname{Pre}(m)\} \operatorname{Body}(m)\{\operatorname{Post}(m)\}$, and if $C$ is a valid configuration, and $C \underset{c}{\longrightarrow} C^{\prime}$, then $C^{\prime}$ is a valid configuration.

\section{Related Work and Conclusions}

In this paper, we have provided formal definitions for both equirecursive and isorecursive interpretations of the kinds of recursive definitions commonly used in static verifiers. We have shown that the isorecursive definitions can be tailored towards practical implementation, while still approximating the equirecursive, which are more suitable for runtime models and soundness proofs. In addition, we have defined a novel Hoare Logic, which assigns a verification semantics to fold and unfold statements explicitly.

The idea of combining separation logic with fractional permissions for concurrent object oriented programming was proposed, and formalised in [4], was adapted to implicit dynamic frames in the form of Chalice[7]. That work was formalised [14] through a Hoare logic for implicit dynamic frames. However, neither [4], nor [14] give a treatment of recursive predicates. A semantics of implicit dynamic frames based on verification condition generation is developed and proven sound in [16].

The first formally rigorous treatment of recursive predicates in the context of separation logics was proposed in [10], where the meaning of predicates was the infinite unrolling of their bodies; the equirecursive treatment. The formal 
approach was further developed in [11]. The tool jStar[3] is based on these ideas, and tries to infer the appropriate fold/unfold steps.

Other implementations, such as, Verifast[6] follow the isorecursive approach, whereby the programmer writes explicit fold/unfold steps. The language is based on separation logic, and does not support heap-dependent expressions. Some other verification tools do not support user-defined predicates, or only support some inbuilt, fixed predicates [1].

The general pattern is clear: equirecursive definitions are employed in formal models, where their intuitive semantics is most appropriate, and is most natural for comparison with a runtime semantics. However, isorecursive definitions are (explicitly or implicitly) used to build most practical implementations of verifiers. The work of this paper bridges this gap, by providing the formal means to compare both semantics, and showing how this difference between an implementation and a formal model can be rigorously justified.

\section{References}

1. J. Berdine, C. Calcagno, and P. W. O'Hearn. Smallfoot: Modular Automatic Assertion Checking with Separation Logic. In FMCO, 2005.

2. J. T. Boyland and W. Retert. Connecting effects and uniqueness with adoption. In POPL, 2005.

3. D. DiStefano and M. J. Parkinson. jStar: Towards practical verification for Java. In OOPSLA. ACM Press, 2008.

4. C. Haack and C. Hurlin. Separation logic contracts for a Java-like language with fork/join. In International Conference on Algebraic Methodology and Software Technology (AMAST'08), July 2008.

5. S. S. Ishtiaq and P. W. O'Hearn. BI as an assertion language for mutable data structures. In POPL, pages 14-26. ACM Press, 2001.

6. B. Jacobs, J. Smans, P. Philippaerts, F. Vogels, W. Penninckx, and F. Piessens. Verifast: A powerful, sound, predictable, fast verifier for $\mathrm{C}$ and Java - invited paper. In $N F M, 2011$.

7. K. R. M. Leino and P. Mueller. Verification of concurrent programs with Chalice. In ESOP, 2009.

8. M. Moskal. Programming with triggers. In SMT '09: Proceedings of the 7th International Workshop on Satisfiability Modulo Theories, 2009.

9. M. Parkinson. Local Reasoning for Java. PhD thesis, University of Cambridge, November 2005.

10. M. Parkinson and G. Bierman. Separation logic and abstraction. In $P O P L$, pages 247-258. ACM Press, 2005.

11. M. Parkinson and G. Bierman. Separation logic, abstraction and inheritance. In POPL, pages 75-86. ACM Press, 2008.

12. M. Parkinson and A. J. Summers. The Relationship between Separation Logic and Implicit Dynamic Frames. In ESOP, 2011.

13. M. Parkinson and A. J. Summers. The Relationship between Separation Logic and Implicit Dynamic Frames. Logical Methods in Computer Science, 2012.

14. A. Raad and S. Srossopoulou. A sip of the chalice. In FTfJP, July 2011.

15. J. Smans, B. Jacobs, and F. Piessens. Implicit dynamic frames: Combining dynamic frames and separation logic. In ECOOP, volume 5653, pages 148-172, July 2009.

16. J. Smans, B. Jacobs, and F. Piessens. Implicit Dynamic Frames. ToPLAS, 2012. 


\section{A Full Definitions, Proofs, Further Lemmas}

\section{A.1 Iso-Semantics}

Definition 8 [Value of Iso-Recursive Expressions] We define evaluation of expressions in a state consisting of heap $H$, and stack frame $\sigma$, through predicate $-\Downarrow_{H, \sigma}$ - as follows :

$$
\begin{array}{lc}
x \Downarrow_{H, \sigma} \sigma(x) \quad \text { null } \Downarrow_{H, \sigma} \text { null } & \text { true } \Downarrow_{H, \sigma} \text { true } \quad \text { false } \Downarrow_{H, \sigma} \text { false } \\
E . f \Downarrow_{H, \sigma} v & \text { if } E . f \Downarrow_{H, \sigma} \iota \text { and } H(\iota, f)=v . \\
E . g\left(E^{\prime}\right) \Downarrow_{H, \sigma} v & \text { if } E \Downarrow_{H, \sigma} \iota \text { and } E \Downarrow_{H, \sigma} v^{\prime} \text { and Body }(g) \Downarrow_{H, \sigma^{\prime}} v, \\
& \text { where } \left.\sigma^{\prime}=\text { [this, } x \mapsto \iota, v\right] . \\
E=E^{\prime} \Downarrow_{H, \sigma} \text { true } & \text { if } E \Downarrow_{H, \sigma} v \text { and } E \Downarrow_{H, \sigma} v \text { for some } v . \\
E=E^{\prime} \Downarrow_{H, \sigma} \text { false } & \text { if } E \Downarrow_{H, \sigma} v \text { and } E \Downarrow_{H, \sigma} v^{\prime} \text { and } v \neq v^{\prime} . \\
\left(E_{1} ? E_{2}: E_{3}\right) \Downarrow_{H, \sigma} v & \text { if } E_{1} \Downarrow_{H, \sigma} \text { true and } E_{2} \Downarrow_{H, \sigma} v . \\
\left(E_{1} ? E_{2}: E_{3}\right) \Downarrow_{H, \sigma} v & \text { if } E_{1} \Downarrow_{H, \sigma} \text { false and } E_{3} \Downarrow_{H, \sigma} v . \\
\text { unfolding } E_{1} . P \text { in } E_{2} \Downarrow_{H, \sigma} v & \text { if } E \Downarrow_{H, \sigma} v
\end{array}
$$

We now define the value of an expression $e$ in the context of heap $H$ as follows:

$$
\|E\|_{H, \sigma}=\left\{\begin{array}{lr}
v & \text { if } E \Downarrow_{H, \sigma} v \\
\text { error }_{t} & \text { if } \nexists v \cdot E \Downarrow_{H, \sigma} v \text { and } \quad E \text { has type } t .
\end{array}\right.
$$

\section{Definition 11 [Framed and Self-Framing iso-assertions]}

An iso-assertion expression $E$, or assertion $A$ is iso-framed in a state consisting of $H, \Pi$ and $\sigma$, written $\models_{\text {frml }}^{\Pi, H, \sigma} E$ and $\models_{\text {frml }}^{\Pi, H, \sigma} A$ respectively, under the following conditions:

$$
\begin{aligned}
& \models_{\text {frml }}^{\Pi, H, \sigma} \text { null } \quad \models_{\text {frml }}^{\Pi, H, \sigma} \text { true } \quad \models_{\text {frml }}^{\Pi, H, \sigma} \text { false } \quad \models_{\text {frml }}^{\Pi, H, \sigma} x \\
& \models_{\text {frml }}^{\Pi, H, \sigma} E . f \quad \Longleftrightarrow \models_{\text {frml }}^{\Pi \Pi, H, \sigma} E \wedge \Pi\left(\|E\|_{H, \sigma}, f\right)>0 \\
& =\stackrel{I, H, \sigma}{I, H} \operatorname{Erml}\left(E^{\prime}\right) \\
& \Longleftrightarrow \models_{\text {frml }}^{I I, H, \sigma} E \wedge \models_{\text {frml }}^{\Pi, H, \sigma} E^{\prime} \wedge \Pi, H, \sigma^{\prime} \models_{\mathrm{I}} \operatorname{Pre}(g) \\
& \text { where } \sigma^{\prime}=\left[\text { this, } \mathrm{x} \mapsto\|E\|_{H, \sigma}, \mid\left[E^{\prime} \|_{H, \sigma}\right]\right. \\
& =_{\text {frml }}^{\Pi, H, \sigma} E=E^{\prime} \\
& ={ }_{\mathrm{frml}}^{I T, H, \sigma} E ? E^{\prime}: E^{\prime \prime} \\
& \Longleftrightarrow \models_{\text {frml }}^{\Pi, H, \sigma} E \wedge \models_{\text {frml }}^{\Pi, H, \sigma} E^{\prime} \\
& \Longleftrightarrow \models_{\mathrm{frml}}^{I, H, \sigma} E \wedge\left(\Pi, H, \sigma \models_{\mathrm{I}} E \Rightarrow \models_{\mathrm{frml}}^{\Pi, H, \sigma} E^{\prime}\right) \\
& \wedge\left(\Pi, H, \sigma \forall_{I} E \Rightarrow \models_{\text {frml }}^{\Pi, H, \sigma} E^{\prime \prime}\right) \\
& \models_{\text {frml }}^{\Pi, H, \sigma} \text { unfolding } E . P \text { in } E^{\prime} \Longleftrightarrow \models_{\text {frml }}^{\Pi, H, \sigma} E \wedge \Pi\left(\|E\|_{H, \sigma}, P\right) \geq 1 \wedge \models_{\text {frml }}^{\Pi^{\prime}, H, \sigma} E^{\prime} \text {, } \\
& \text { where } \Pi^{\prime}=\Pi \odot_{H}\|E\|_{H, \sigma} . P \sigma \\
& \models_{\mathrm{frml}}^{\Pi, H, \sigma} \operatorname{acc}(E . P, q) \quad \Longleftrightarrow \models_{\mathrm{frml}}^{\Pi, H, \sigma} E \\
& =\stackrel{\Pi=, H, \sigma}{=} E \rightarrow A \\
& =_{\text {frml }}^{I I, H, \sigma} A_{1} * A_{2} \\
& \begin{array}{l}
\Longleftrightarrow \models_{\mathrm{frml}}^{I, T} E \\
\Longleftrightarrow \models_{I, H, \sigma} E \wedge\left(\Pi, H, \sigma \models_{I, H} E \Rightarrow=_{\mathrm{frml}}^{\Pi, H, \sigma} E^{\prime}\right)
\end{array}
\end{aligned}
$$

An iso-assertion $A$ is self-framing, written $\models_{\text {frml }} A$ if, for all $H, \Pi$ and $\sigma$ : $\Pi, H, \sigma \models_{\mathrm{I}} A \Rightarrow \models_{\text {frml }}^{\Pi, H, \sigma} A$

\section{A.2 Comparing the Assertion Semantics}

\section{Definition ??[Encoding iso-assertions to equi-assertions]}


We now define the encoding $\langle\langle-\rangle\rangle$ which maps isorecursive expressions and statements to their equirecursive counterparts:

$$
\begin{aligned}
\langle\langle\text { null }\rangle\rangle & =\text { null } \\
\langle\langle\text { false }\rangle & =\text { false } \\
\langle\langle\text { true }\rangle\rangle & =\text { true } \\
\langle\langle x\rangle\rangle & =x \\
\langle\langle E . f\rangle\rangle & =\langle\langle E\rangle . f \\
\langle E E 1=E 2\rangle\rangle & =\langle\langle E 1\rangle\rangle=\langle\langle E 2\rangle\rangle \\
\left\langle\left\langle E . g\left(E^{\prime}\right)\right\rangle\right\rangle & =\left\langle\langle E\rangle . g\left(\left\langle\left\langle E^{\prime}\right\rangle\right\rangle\right)\right. \\
\left\langle\left\langle E ? E^{\prime}: E^{\prime \prime}\right\rangle\right\rangle & \left.=\langle\langle E\rangle\rangle ?\left\langle E^{\prime}\right\rangle\right\rangle:\left\langle\left\langle E^{\prime \prime}\right\rangle\right\rangle \\
\left\langle\left\langle\text { unfolding } E . P \operatorname{in} E^{\prime}\right\rangle\right\rangle & =\left\langle\left\langle E^{\prime}\right\rangle\right\rangle \\
\langle\langle\operatorname{acc}(E . f, q)\rangle\rangle & =\operatorname{acc}(E . f, q) \\
\langle\langle\operatorname{acc}(E . P)\rangle\rangle & =\langle\langle E\rangle . P \\
\left\langle\left\langle A * A^{\prime}\right\rangle\right\rangle & =\langle\langle A\rangle\rangle *\left\langle\left\langle A^{\prime}\right\rangle\right\rangle \\
\langle\langle E \rightarrow A\rangle\rangle & =\langle\langle E\rangle \rightarrow\langle\langle A\rangle\rangle
\end{aligned}
$$

Lemma 1 In a well-defined program,

1. If $\Pi, H, \sigma$ is a good iso-state, and $\Pi(\iota . P)\rangle 0$, then $\left\langle\langle\Pi\rangle_{H}=\left\langle\left\langle\Pi \odot_{H} \iota . P\right\rangle_{H}\right.\right.$.

2. If $\pi, H, \sigma \models_{\mathrm{E}}\langle\langle A\rangle\rangle$, then $\exists \Pi$ s.t. $(\Pi, H, \sigma)$ is a good iso-state, $\langle\langle\Pi\rangle\rangle_{H}=\pi$, and $\pi, H, \sigma|=| A$.

Proof. Part 1, by direct application of the definitions. Part 2 follows by induction on the structure of $A$; when $A$ is an $E$, using Theorem 1.1.

\section{Theorem 1}

1. $\|\left\langle\langle E\rangle\left\|_{H, \sigma}=\right\| E \|_{H, \sigma}\right.$.

2. If $\Pi, H, \sigma \models_{1} A$, and $(\Pi, H, \sigma)$ is a good iso-state, then $\left\langle\langle\Pi\rangle_{H}, H, \sigma \models_{\mathrm{E}}\right.$ $\langle\langle A\rangle$.

3. If $(\Pi, H, \sigma)$ is a good iso-state, and $\models_{\text {frml }}^{\Pi, H, \sigma} A$, then $\models_{\text {frmE }}^{\langle\langle\Pi\rangle, H, \sigma}\langle\langle A\rangle\rangle$.

4. If all functions and predicates are well-formed, then if $A$ is iso-self-framing, then $\langle\langle A\rangle$ is equi-self-framing

5. If $A \models_{1} A^{\prime}$, then $\left\langle\langle A\rangle \models_{\mathrm{E}}\left\langle\left\langle A^{\prime}\right\rangle\right\rangle\right.$.

Proof. Part 1 by showing that termination of $E$ or $\langle\langle E\rangle\rangle$ implies that $\|\left\langle\langle E\rangle \|_{H, \sigma}=\right.$ $\mid\left[E \|_{H, \sigma}\right.$ - this is by induction on the derivation of $-\Downarrow_{H, \sigma}$. This results also gives that the term $E$ loops forever if and only if $\langle\langle E\rangle\rangle$ loops forever. Part 2 by induction on the structure of $A$.

Part 3 follows by induction on $A$, and requires an analogous lemma for expressions.

Part 4, follows from Lemma 1 and part 3.

For Part 5, take any $H, H^{\prime}, \pi, \sigma$, st: $\pi, H, \sigma \models_{\mathrm{E}}\langle\langle A\rangle$. We want to show that $\pi, H, \sigma \models_{\mathrm{E}}\left\langle\left\langle A^{\prime}\right\rangle\right\rangle$. Because we have $\pi, H, \sigma \models_{\mathrm{E}}\langle\langle A\rangle\rangle$, by application of Lemma 1, we obtain that there exists a $\Pi$, s.t. $\langle\langle\Pi\rangle\rangle_{H}=\pi$, and $H \models \Pi$, and $\Pi, H, \sigma \models_{\mathrm{I}} A$. By the assumption, we obtain that $\Pi, H, \sigma \models_{\mathrm{I}} A^{\prime}$. This, together with part 2 gives that $\left\langle\langle\Pi\rangle_{H}, H, \sigma=_{\mathrm{E}}\left\langle\left\langle A^{\prime}\right\rangle\right\rangle\right.$. 


\section{A.3 Isorecursive Hoare Logic}

\section{Lemma 2 [Generation Lemma]}

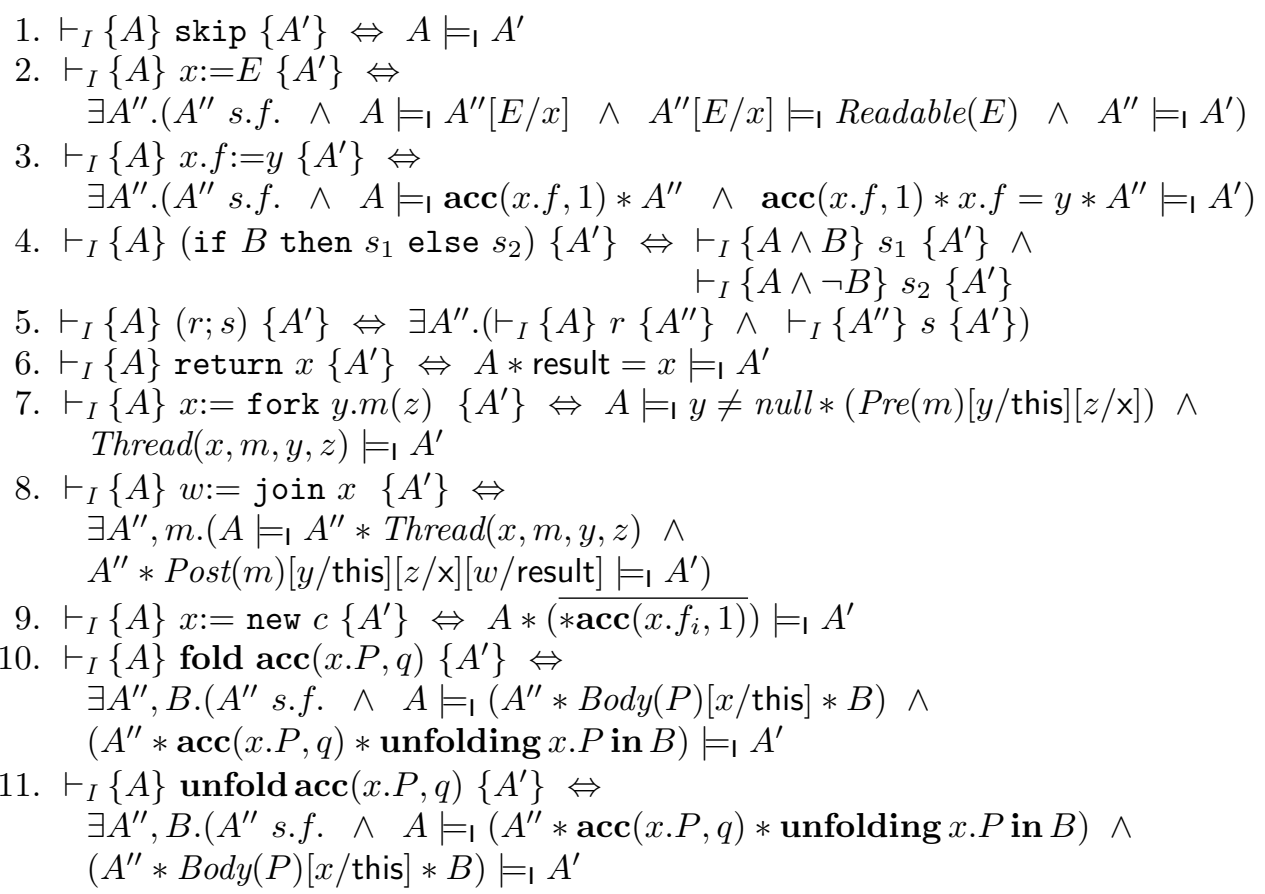

\section{A.4 Equirecursive Hoare Logic}

(See Figure 4)

\section{A.5 Operational Semantics}

Theorem 3 [Soundness of Verification] For a well-formed program, if all method definitions satisfy $\vdash_{I}\{\operatorname{Pre}(m)\} \operatorname{Body}(m)\{\operatorname{Post}(m)\}$, and if $C$ is a valid configuration, and $C \underset{c}{\longrightarrow} C^{\prime}$, then $C^{\prime}$ is a valid configuration.

Proof. Sketch: By induction on the length of the execution $C \longrightarrow C^{\prime}$. The proof relies on three main points. Firstly, for every local and paired transition, the corresponding Hoare triple never requires more permission in the postcondition than in the precondition. Secondly, for every such transition, a heap location is only modified if full permission to the location was required in the precondition of the corresponding triple. Thirdly, since all pre-conditions are self-framing, the preconditions of all other entities remain unaffected by these modifications. This relies on the (easy) lemma that, if $\pi, H, \sigma \models_{\mathrm{E}} a$ holds, and $a$ is self-framing, then in a heap $H^{\prime}$ which agrees with $H$ on at least the values of locations to which $\pi$ requires permission, $\pi, H^{\prime}, \sigma \models_{\mathrm{E}} a$ also holds (this is sometimes called stability of assertions. 


$$
\begin{aligned}
& \overline{\vdash_{E}\{a\} \operatorname{skip}\{a\}}(\operatorname{skip} E) \\
& \frac{a[e / x] \models_{1} \text { Readable }(e)}{\vdash_{E}\{a[e / x]\} x:=e\{a\}}(\text { varassE) } \\
& \overline{\vdash_{E}\{x \neq n u l l * \operatorname{acc}(x . f, 1)\} x . f:=y\{\operatorname{acc}(x . f, 1) * x . f=y\}} \text { (fldassE) } \\
& \overline{\vdash_{E}\{a\} \text { return } x\{a * \text { result }=x\}}(\operatorname{ret} E) \\
& \frac{\overline{f_{i}}=\text { fields }(c)}{\overline{\vdash_{E}\{\text { true }\}} x:=\text { new } c\left\{\left(\overline{* \operatorname{acc}\left(x . f_{i}, 1\right)}\right)\right\}}(\text { newE }) \\
& \frac{\vdash_{E}\{a * b\} s_{1}\left\{a^{\prime}\right\} \quad \vdash_{E}\{a * \neg b\} s_{2}\left\{a^{\prime}\right\}}{\vdash_{E}\{a\}\left(\text { if } b \text { then } s_{1} \text { else } s_{2}\right)\left\{a^{\prime}\right\}}(\text { if } E) \\
& \frac{a=\operatorname{Pre}(m)[y / \text { this }][z / \mathrm{x}]}{\vdash_{E}\{y \neq n u l l * a\} \quad x:=\text { fork } y \cdot m(z)\{\operatorname{Thread}(x, m, y, z)\}}(\text { forkE }) \\
& \frac{a^{\prime}=\operatorname{Post}(m)[y / \text { this }][z / \mathrm{x}][w / \text { result }]}{\vdash_{E}\{\operatorname{Thread}(x, m, y, z)\} w:=\text { join } x \quad\left\{a^{\prime}\right\}}(\text { joinE }) \\
& \frac{\vdash_{E}\{a\} r\left\{a^{\prime}\right\} \quad \vdash_{E}\left\{a^{\prime}\right\} s\left\{a^{\prime \prime}\right\}}{\vdash_{E}\{a\}(r ; s)\left\{a^{\prime \prime}\right\}}(\operatorname{seqE}) \\
& \frac{a_{1} \models_{1} a_{3} \quad \vdash_{E}\left\{a_{3}\right\} s\left\{a_{4}\right\} \quad a_{4} \models_{1} a_{2}}{\vdash_{E}\left\{a_{1}\right\} s\left\{a_{2}\right\}}(\text { consE }) \\
& \frac{\vdash_{E}\{a\} s\left\{a^{\prime}\right\} \quad a^{\prime \prime} s . f . \quad \bmod (s) \cap F V\left(a^{\prime \prime}\right)=\emptyset}{\vdash_{E}\left\{a^{\prime \prime} * a\right\} s\left\{a^{\prime} * a^{\prime \prime}\right\}} \text { (frameE) }
\end{aligned}
$$

Fig. 4. Hoare Logic for Equirecursive semantics 


$$
\begin{gathered}
\left.\frac{h,\{\sigma,(\text { skip } ; s)\}_{t} \rightarrow h,\{\sigma, s\}_{t}}{l} \text { (skipS }\right) \\
\frac{\|e\|_{h, \sigma}=v}{h,\{\sigma,(x:=e ; s)\}_{t} \longrightarrow h,\{\sigma[x \mapsto v], s\}_{t}}(\text { varass } S) \\
\frac{\sigma(x)=\iota \quad h^{\prime}=h[(\iota, f) \mapsto \sigma(y)]}{h,\{\sigma,(x \cdot f:=y ; s)\}_{t} \longrightarrow h^{\prime},\{\sigma, s\}_{t}}(\text { fldass } S) \\
\left.\frac{\|b\|_{h, \sigma}=\text { true }}{h,\left\{\sigma,\left(\left(\text { if } b \text { then } s_{1} \text { else } s_{2}\right) ; s_{3}\right)\right\}_{t} \longrightarrow h,\left\{\sigma,\left(s_{1} ; s_{3}\right)\right\}_{t}} \text { (iftrueS }\right) \\
\left.\frac{\|b\|_{h, \sigma}=f a l s e}{h,\left\{\sigma,\left(\left(\text { if } b \text { then } s_{1} \text { else } s_{2}\right) ; s_{3}\right)\right\}_{t} \rightarrow h,\left\{\sigma,\left(s_{2} ; s_{3}\right)\right\}_{t}} \text { (iffalse } S\right)
\end{gathered}
$$

Fig. 5. Local transitions 